\title{
QUALIDADE DE VIDA RELACIONADA À SAÚDE DE PACIENTES COM CÂNCER DE PRÓSTATA EM TRATAMENTO DE RADIOTERAPIA: REVISÃO INTEGRATIVA DE LITERATURA
}

\author{
Patrícia Daniela dos Santos Quijada ${ }^{1}$ \\ Paolla Algarte Fernandes ${ }^{2}$ \\ Branca Maria de Oliveira Santos ${ }^{3}$
}

QUIJADA, P. D. dos S.; FERNANDES, P. A.; SANTOS, B. M. de O. Qualidade de vida relacionada à saúde de pacientes com câncer de próstata em tratamento de radioterapia: revisão integrativa de literatura. Arq. Cienc. Saúde UNIPAR, Umuarama, v. 22, n. 3, p. 199-204, set./dez. 2018.

RESUMO: O presente estudo objetiva analisar as publicações científicas de alto impacto que avaliaram a qualidade de vida relacionada à saúde de pacientes com câncer de próstata em tratamento radioterápico. Para tanto, ancora-se num estudo exploratório, de revisão integrativa da literatura, utilizando as bases de dados PubMed, Web of Science, Scopus e Lilacs, com os descritores: Quality of life, Prostatic neoplasms e Radiotherapy, usando o operador booleano AND. Do total de 1418 artigos localizados, foram excluídos 1403 após o uso dos filtros adotados como critérios de inclusão e exclusão, de acordo com cada base de dados, e leitura dos respectivos resumos, resultando em 15 artigos. Os resultados evidenciaram que o tratamento de radioterapia causa impacto na qualidade de vida relacionada à saúde dos pacientes, com manifestações de sinais e sintomas de intensidade variada, principalmente nas funções urinária, sexual e intestinal. Mesmo havendo declínio na qualidade de vida relacionada à saúde durante o tratamento, os efeitos negativos foram limitados e temporários, podendo melhorar com o passar do tempo. Ainda que os estudos tenham sido realizados em momentos e países diferentes, com amostras, modalidades de radiação e instrumentos distintos, os resultados contribuíram para o planejamento, a execução e avaliação do tratamento radioterápico no cotidiano dos serviços oncológicos, considerando a realidade de cada um. Recomenda-se a realização de novas pesquisas de modo a possibilitar a identificação dos efeitos dos diferentes tipos de radiação e suas consequências na vida dos pacientes, com vistas à escolha de medidas terapêuticas menos impactantes.

PALAVRAS-CHAVE: Câncer de próstata. Qualidade de vida. Radioterapia.

\section{QUALITY OF LIFE OF PROSTATE CANCER PATIENTS UNDERGOING RADIATION TREATMENT: AN INTEGRATIVE LITERATURE REVIEW}

\begin{abstract}
This paper has the aim of reviewing evidence on quality of life aspects related to the health of prostate cancer patients undergoing radiotherapy. An exploratory study was developed with an integrative literature review using the PubMed, Web of Science, Scopus, and Lilacs websites with the key words: Quality of life, Prostatic neoplasms, and Radiotherapy, using the Boolean AND operator. From the total 1418 articles found, 1403 were excluded after the use of filters adopted as inclusion and exclusion criteria, according to each database, and the reading of the abstracts, resulting in 15 articles. The results showed that the radiotherapy treatment has impact on the quality of life related to the health of patients with signs of manifestations and symptoms of varying intensity, especially in the urinary, sexual and bowel functions. Even though there is a decline in the quality of life related to health during the treatment, the negative effects were limited and temporary, meaning they may improve over time. Although the studies were carried out at different times and in different countries, with different number of samples, radiation modes and instruments, the results contributed to the planning, implementation and assessment of radiotherapy treatment in the routine of cancer services, considering their realities. Further research is recommended to enable the identification of the effects of different types of radiation therapy and their consequences in the lives of patients, with a view to choosing less impactful therapeutic measures.
\end{abstract}

KEYWORDS: Prostatic neoplasms. Quality of life. Radiotherapy.

\section{Introdução}

Atualmente, o câncer é um dos problemas de saúde pública mais complexos que o sistema de saúde brasileiro enfrenta, dada a sua magnitude epidemiológica, social e econômica (HERR et al., 2013). Estimativas mundiais apontam 14,1 milhões de casos novos de câncer e um total de 8,2 milhões de mortes em todo o mundo, em 2012 (FERLAY et al., 2015). No Brasil, para o ano de 2016/2017, a ocorrência esperada é de aproximadamente 600 mil casos novos (BRASIL, 2015).

O câncer de próstata $(\mathrm{CaP})$ é considerado o segundo tipo mais comum em homens, com cerca de 1,1 milhão de casos diagnosticados em todo mundo no ano de 2012, dos quais 70\% em países desenvolvidos (FERLAY et al., 2015). No Brasil, a estimativa em 2016/2017 é de 61.200 casos novos para cada 100 mil homens. Sem considerar os tumores de pele não melanoma, o CaP é o mais incidente entre os homens em todas as regiões do país (BRASIL, 2015).

$\mathrm{O}$ único fator de risco bem estabelecido para o desenvolvimento do CaP é a idade. Cerca de $62 \%$ dos casos diagnosticados no mundo ocorrem em homens com 65 anos ou mais. Além disso, a dieta, a etnia e a história familiar também são considerados importantes fatores de risco (BRASIL, 2015).

No momento em que o paciente recebe o diagnóstico de CaP, obtido com a utilização de técnicas histológicas define-se o tipo de tratamento e o possível prognóstico da do-

DOI: 10.25110 /arqsaude.v22i3.2018.5835

${ }^{1}$ Enfermeira, Discente do Programa de Doutorado em Promoção de Saúde - Universidade de Franca, Franca-SP, Brasil. E-mail:patriciaquijadatizzo@gmail. com

${ }^{2}$ Enfermeira, Discente do Programa de Mestrado em Promoção de Saúde - Universidade de Franca, Franca-SP, Brasil.

${ }^{3}$ Enfermeira, Livre-Docente em Enfermagem -USP. Docente do Programa de Mestrado e Doutorado em Promoção de Saúde - Universidade de Franca, Franca-SP, Brasil. 
ença. As opções para o tratamento visam não somente o controle oncológico como também a manutenção da qualidade de vida relacionada à saúde (QVRS) (DAMIÃO et al., 2015).

Dentre as opções de tratamento, a radioterapia, geralmente, é indicada nos casos de tumores localizados, sendo uma terapêutica local, com aplicação delimitada e específica, podendo ser utilizada de forma isolada ou em combinação com outro tratamento (SALVAJOLI; SALVAJOLI, 2012). Ainda que mantenha a integridade dos órgãos, os pacientes podem experimentar inúmeros efeitos colaterais ou reações adversas, comuns às diferentes modalidades de radioterapia, como os sintomas miccionais irritativos, proctite em $70 \%$ a $80 \%$ dos casos, retenção urinária e disfunção erétil (em 45\% dos pacientes), que comprometem gradualmente a QVRS (PAULA; SAWADA, 2015; SCIARRA et al., 2013).

Os efeitos colaterais da radioterapia faz sobressair a importância da avaliação da QVRS dos pacientes ao longo do tratamento, de modo a oferecer subsídios para o acompanhamento multidisciplinar na prestação da assistência, com vistas a amenizar os sintomas e a garantir boa aderência do paciente ao tratamento (PAULA; SAWADA, 2015). Sua avaliação possibilitará o conhecimento global dos efeitos da doença e das terapêuticas instituídas com intuito curativo, pois permite avaliar qual a percepção que o paciente tem dos efeitos somático e psicossocial induzidos pelo tratamento (CRAMER; SPILKER, 1998).

O conhecimento da QVRS pode fornecer dados para uma tomada de decisão mais racional, quer para o indivíduo, quer para uma determinada população. A avaliação da QRVS de pacientes com longa sobrevivência, após terem sido curados de uma neoplasia, permite obter informações que podem melhorar os procedimentos terapêuticos para utilização em outros pacientes com a mesma patologia (JENNEY, 1996; GREIL et al., 1999).

Assim, o objetivo deste estudo foi realizar uma revisão integrativa da literatura, com vistas a analisar a qualidade de vida relacionada à saúde de pacientes com câncer de próstata em tratamento radioterápico.

\section{Método}

A revisão integrativa é recurso metodológico que permite reunir e sistematizar resultados de estudos significativos sobre um tema específico, com a finalidade de aumentar e fortalecer o conhecimento científico de determinadas áreas e para auxiliar a tomada de decisões dos profissionais (SOUZA; SILVA; CARVALHO, 2010).

Para a realização deste estudo optou-se pela referida revisão seguindo seis etapas: identificação do tema e da questão norteadora da pesquisa, definição dos critérios de inclusão e exclusão, informações a serem analisadas, categorização e avaliação dos estudos, interpretação dos resultados e conclusão (TEIXEIRA et al., 2013).

Para guiar a presente revisão, formulou-se a seguinte questão norteadora: "Quais as evidências sobre os aspectos da qualidade de vida relacionada à saúde de pacientes com câncer de próstata em tratamento radioterápico nas literaturas nacionais e internacionais?

As publicações foram identificadas por meio de palavras-chaves, Descritores em Ciência da Saúde (DeCS) e MeSH (Medical Subject Headings): quality of life, prostatic neoplasms e radiotherapy, conforme solicitado em cada base, usando o operador booleano AND, de modo a contemplar todas as palavras utilizadas para a busca. A seleção dos artigos ocorreu nos meses de setembro a novembro de 2015, nas bases National Library of Medicine National Institutes of Health (MEDLINE/PubMed), Literatura Latino-Americana e do Caribe em Ciências da Saúde (Lilacs), Web of Science e Scopus. Considerando a utilização de bases diferentes levou-se, inicialmente, em consideração, a necessidade de filtros de busca validados por cada base de dados selecionada para se chegar à seleção dos artigos identificados em cada uma delas. Assim, após a seleção das 1.273 publicações obtidas na primeira busca, realizada na base de dados eletrônica PubMed (Medical Literature Analysis and Retrieval System Online) com os descritos do MeSH e a aplicação dos filtros adotados pela referida base de dados (article types, text avaliability, publication dates, species) resultaram em 109 artigos. Desses, após leitura dos resumos, foram excluídos 31 que tratavam-se de validação de instrumento e outros tratamentos e 57 por não atenderem ao objetivo da revisão proposta, resultando em 21 artigos para leitura na íntegra, dos quais foram selecionados 10 para compor a revisão.

$\mathrm{Na}$ segunda busca, realizada na biblioteca virtual LILACS (Literatura científica e técnica da América Latina e Caribe/BVS-Biblioteca Virtual em Saúde) utilizando os descritores do DeCS, encontraram-se 10 artigos, que após aplicação dos filtros (texto completo, idiomas, ano de publicação, tipo de documento) resultaram em três para leitura dos resumos, finalizando com um artigo para leitura na íntegra, que após a leitura foi excluído por não atender ao objetivo proposto.

$\mathrm{Na}$ terceira busca, realizada na base de dados Web of Sicence, com as referidas palavras-chaves, encontraram-se 120 artigos, que após aplicação do filtro refine results (article, languages, publications years), resultaram em 42 artigos para leitura dos resumos, culminando na exclusão de 32 que estavam duplicados na base de dados PubMed. Após a leitura dos 10 artigos na íntegra, foram selecionados cinco para compor a revisão. Na quarta busca, na base de dados Scopus, utilizando as palavras-chaves, foram identificados 15 artigos, que após aplicação do filtro refine results (year, document type, languages), resultaram em dois artigos. Pela leitura dos resumos, foi selecionado um artigo para leitura na íntegra e excluído por não atender ao objetivo.

Os critérios de inclusão adotados para seleção da amostra foram: artigos publicados em periódicos nacionais e internacionais, indexados no período entre 2010 a 2015 , disponíveis na íntegra e nos idiomas português, inglês e espanhol; revisados por pares e que abordassem o tema proposto, acerca do desfecho da QVRS de pacientes com $\mathrm{CaP}$ submetidos ao tratamento de radioterapia. Os critérios de exclusão adotados foram: artigos que objetivavam a criação e/ou validação de instrumentos de qualidade de vida (QV) e aqueles que relacionavam a radioterapia a outras modalidades terapêuticas.

Com o uso dos descritores foram localizados nas bases 1418 artigos, dos quais 1273 na PubMed, 120 na Web of Science, 15 na Scopus e 10 na Lilacs. Com o uso dos filtros adotados como critérios de inclusão e exclusão, de acordo com cada base de dados, foram excluídos 1262 artigos, restando 156. Após a leitura dos respectivos resumos, foram 
excluídos 74 por estarem duplicados nas bases PubMed, Web of Science e Scopus e 49 por tratarem de estudos de validação de instrumentos ou que relacionavam a radioterapia a outras modalidades terapêuticas. Em seguida, após leitura minuciosa dos artigos na íntegra, foram excluídos mais 18 artigos, por não atenderem ao objetivo proposto usando outras modalidades terapêuticas, terminando com o total de 15 artigos. A figura 1 apresenta o fluxograma utilizado para a seleção das publicações por etapas.

1. Fluxograma utilizado para a seleção das publicações por etapas e seus respectivos resultados

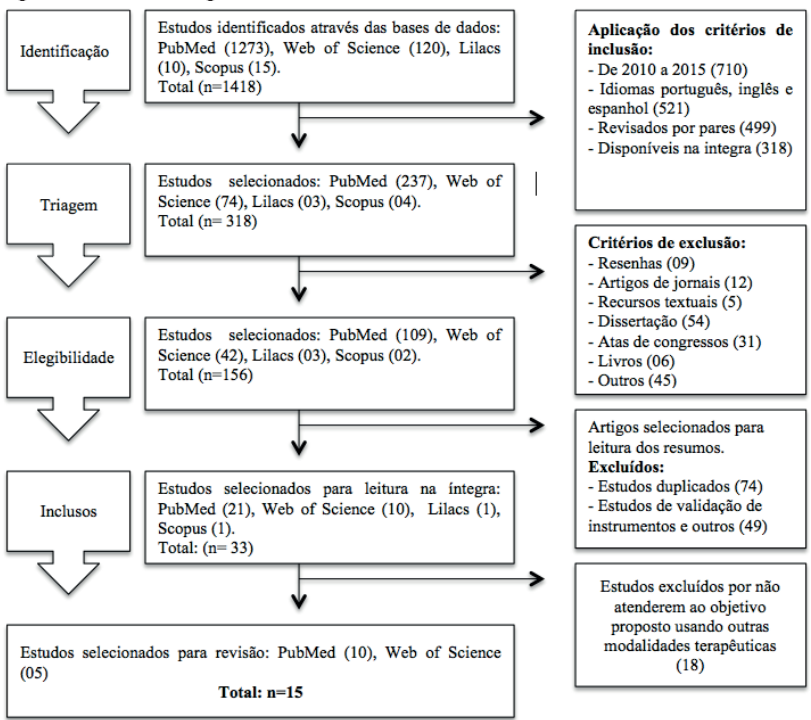

Para análise dos artigos foi desenvolvido um formulário de coleta de dados baseado na literatura contendo os seguintes itens: títulos dos artigos, autores, ano, país, idiomas, objetivos, delineamentos, resultados, conclusões (MELNYK; FINEOUT-OVERHOLT, 2011).

\section{Resultados}

Inicialmente, vale considerar, que dos 15 artigos incluídos na revisão, 5 (33,3\%) foram publicados no ano de 2010, 4 (26,6\%) em 2012, 2 (13,3\%) em 2011, 2013 e 2014, respectivamente. Em relação ao país de origem, observou-se a liderança dos Estados Unidos, com 7 (46,6\%) publicações. A seguir vieram a Alemanha, o Canadá e a Holanda, com duas (13,3\%) publicações cada um e a Lituânia e o Japão, com uma publicação $(6,6 \%)$ cada. Todos foram publicados em inglês.

Os objetivos dos estudos, estavam claramente definidos em relação ao foco e abrangência, viabilizando a ação pretendida. Eles foram divididos e agrupados de acordo com o tipo de avaliação da QVRS de cada estudo. Dos estudos, seis avaliaram a QVRS em modalidades específicas de radiação: terapia de prótons, radioterapia por feixe externo, radioterapia de intensidade modulada, radioterapia conformada e radioterapia estereotáxica (Quadro 1), cinco avaliaram os efeitos dos sinais e sintomas e a repercussão do tratamento na QVRS (Quadro 2) e quatro avaliaram com foco na comparação entre modalidades de radiação e de alterações de aumento das doses de radiação: radioterapia de intensidade modulada/radioterapia guiada por imagem, radioterapia de intensidade modulada/radioterapia conformada convencional, hipofracionada/radioterapia de intensidade modulada (Quadro 3). Nos quadros é possível visualizar a síntese dos objetivos, delineamentos, amostras, instrumentos, resultados e conclusões dos estudos.

Pela análise dos delineamentos observou-se que todos foram realizados em hospitais específicos para tratamento oncológico, com número variável de participantes; as entrevistas foram realizadas, na maioria, antes, durante e até 36 meses após o tratamento, com diferentes tipos de instrumentos de avaliação de $\mathrm{QV}$, de forma individual ou em conjunto de até três instrumentos diferentes; 10 (66,6\%) estudos foram classificados pelos autores como prospectivos, 2 $(13,3 \%)$ como randomizados e um $(6,6 \%)$ como descritivo, transversal e coorte, respectivamente.

Pelos resultados e conclusões dos estudos, a maioria identificou impacto negativo na QVRS durante o tratamento de radioterapia, principalmente nas funções sexuais, urinárias e intestinais. Os estudos que avaliaram a QVRS antes, durante e após o tratamento, demonstraram melhora significativa na QVRS, em média, após dois anos do término do tratamento.

Quadro 1: Síntese dos estudos que avaliaram a QVRS em modalidades específicas de radiação.

\begin{tabular}{|c|c|c|}
\hline Objetivos & $\begin{array}{c}\text { Delineamentos/ } \\
\text { Amostras e } \\
\text { Instrumentos }\end{array}$ & Resultados/Conclusões \\
\hline $\begin{array}{l}\text { Avaliar, prospectivamente } \\
\text { a QV de pacientes que } \\
\text { receberam radioterapia } \\
\text { conformada (GEINITZ et } \\
\text { al., 2010) }\end{array}$ & $\begin{array}{c}\text { Prospectivo } \\
78 \\
\text { EORTC QLQ C30 }\end{array}$ & $\begin{array}{l}\text { Os efeitos da radioterapia conformada na QV foram } \\
\text { limitados e, principalmente, temporários. Os sintomas } \\
\text { urinários foram afetados, mas recuperaram dentro de } 8 \\
\text { semanas a um ano após a radioterapia. Devido ao seu } \\
\text { pequeno impacto negativo na QVRS, a radioterapia } \\
\text { conformada para o CaP pode ser um excelente } \\
\text { tratamento. }\end{array}$ \\
\hline $\begin{array}{l}\text { Avaliar o impacto sobre a } \\
\text { QV de pacientes tratados } \\
\text { com radioterapia } \\
\text { estereot áxica (KATZ et al., } \\
\text { 2010). }\end{array}$ & $\begin{array}{c}\text { Prospectivo } \\
304 \\
\text { EPIC }\end{array}$ & $\begin{array}{l}\text { Os pacientes foram acompanhados em média durante } \\
30 \text { meses. Os domínios das funçõos urinárias e } \\
\text { intest inais diminuiram no início do tratamento } \\
\text { voltaram aos seus valores normais. Na função sexual } \\
\text { houve uma queda de } 10 \% \text { para todos os pacientes. Em } \\
\text { longo prazo, seguimentos e estudos adicionais são } \\
\text { necessários para confirmar a durabilidade do controle } \\
\text { bioquímico, toxicidade e QV de pacientes tratados com } \\
\text { radioterapia estereotáxica. }\end{array}$ \\
\hline $\begin{array}{l}\text { Avaliar a QV após a } \\
\text { terapia de prótons para o } \\
\text { CaP em homens com até } \\
60 \text { anos (HOPPE et al., } \\
\text { 2012) }\end{array}$ & $\begin{array}{c}\text { Prospectivo } \\
262 \\
\text { EPIC } \\
\text { International Index } \\
\text { of Erectile Func tion }\end{array}$ & $\begin{array}{l}\text { O maior declinio na pontuação sexual ocorreu no no } \\
\text { primeiro ano após o tratamento. A atividade sexual } \\
\text { caiu } 11 \% \text { em dois anos. Os jovens submetidos à terapia } \\
\text { de prótons tiveram excelentes resultados em relação à } \\
\text { disfunção erétil, incontinência urinária e outros } \\
\text { parâmetros de QV durante os primeiros dois anos após } \\
\text { o tratamento. A terapia de prótons pode oferecer aos } \\
\text { jovens uma excelente opção de tratamento, com menor } \\
\text { risco de disfunção erétil e incontinência urinária. }\end{array}$ \\
\hline $\begin{array}{l}\text { Investigar a QV dos } \\
\text { pacientes tratados com } \\
\text { radiot erapia por feixe } \\
\text { externo e comparar os } \\
\text { resultados com uma } \\
\text { população normal } \\
\text { (SCHAAKE et al.,2013). }\end{array}$ & $\begin{array}{c}\text { Prospectivo } \\
227 \\
\text { EORTC QLQ C30 }\end{array}$ & $\begin{array}{l}\text { Uma redução mínima, mas estatisticamente } \\
\text { significa nte, foi observada para o funcionamento } \\
\text { fisico. Os pacientes com doençaa coronária/pulmonar } \\
\text { obstrutiva crôn ica ou asma tiveram um curso } \\
\text { significativamente pior na QV. Fadiga, dispneia e } \\
\text { insónia aumentaram após o tratamento com os niveis } \\
\text { máximos de compromet imento em seis meses após o o } \\
\text { tratamento. OO nivel de constipação e diarreia após a } \\
\text { radioterapia aumentou um ano após a radioterapia. }\end{array}$ \\
\hline $\begin{array}{l}\text { Avaliar as mudanças na } \\
\text { QV após a radioterapia de } \\
\text { intensidade modulada em } \\
\text { pacientes com CaP } \\
\text { local izado (YAMAMOTO } \\
\text { et al, 2014). }\end{array}$ & $\begin{array}{c}\text { Prospectivo } \\
91 \\
\text { EPIC }\end{array}$ & $\begin{array}{l}\text { Funções urinárias, incluindo sintomas } \\
\text { irritat ivos/obstrução, e funçôes hormonais não foram } \\
\text { afetadas pela radioterapia de modulada, mas a função } \\
\text { intestinal e o funcionamento sexual diminuiram. A } \\
\text { avaliação do impacto do tratamento na QV foi uma } \\
\text { importante ferramenta para a continuidade do } \\
\text { tratamento de intens idade modulada. }\end{array}$ \\
\hline $\begin{array}{l}\text { Avaliar a toxicidade tardia } \\
\mathrm{e} \mathrm{a} \mathrm{QV} \mathrm{em} \mathrm{pacientes} \mathrm{que} \\
\text { receberam radioterapia de } \\
\text { intensidade modulada } \\
\text { (CHENNUPATI et al, } \\
\text { 2014). }\end{array}$ & $\begin{array}{c}\text { Prospectivo } \\
372 \\
\text { EPIC }\end{array}$ & $\begin{array}{l}\text { Todos os domínios da funcão intestinal foram } \\
\text { negat ivamente afetados em dois anos, com 11\% dos } \\
\text { pacie ntes relatando um moderado ou grave problema } \\
\text { intest inal. Depois da radioterapia de intensidade } \\
\text { modulada, as taxas de toxicidade grave foram baixas, a } \\
\text { gastroi ntestinal e geniturinária permaneceram elevadas. } \\
\text { Esses resultados apoiam o uso de diretrizes estritas de } \\
\text { poupadores retais ao planejarem a radioterapia de } \\
\text { intens idade modulada. }\end{array}$ \\
\hline
\end{tabular}


Quadro 2: Síntese dos estudos que avaliaram os efeitos dos sinais e sintomas e a repercussão do tratamento na QVRS.

\begin{tabular}{|c|c|c|}
\hline Objetivos & $\begin{array}{c}\text { Delineamentos/ } \\
\text { Amostras e } \\
\text { Instrumentos }\end{array}$ & Resultados/Conclusões \\
\hline $\begin{array}{l}\text { Descrever as } \\
\text { alter ações na função } \\
\text { sexual durante a } \\
\text { radioterapia e avaliar } \\
\text { as diferenças de } \\
\text { características } \\
\text { clínicas, estados de } \\
\text { humor e QV } \\
\text { (HOWLETT et al., } \\
\text { 2010). }\end{array}$ & $\begin{array}{l}\text { Descritivo } \\
\quad 70 \\
\text { Questionário de auto } \\
\text { relato }\end{array}$ & $\begin{array}{l}\text { No início e ao final da radiação, cerca de } 50 \% \text { relataram } \\
\text { problemas com a função sexual. No geral, os homens sem } \\
\text { problemas sexuais, tanto no início e ao final da radioterapia, } \\
\text { tiveram significativamente menos ansiedade e depressão. } \\
\text { Conclui que alterações na função sexual durante a radiação } \\
\text { afeta o humor dos pacientes e a QVRS. }\end{array}$ \\
\hline $\begin{array}{l}\text { Esclarecer as relações } \\
\text { entre QV, apoio social } \\
\text { estrutural e funci onal } \\
\text { em homens trat ados } \\
\text { com radioterapia } \\
\text { (QUEENAN et al., } \\
\text { 2010). }\end{array}$ & $\begin{array}{c}\text { Transversal } \\
155 \\
\text { EORTC QLQ C30 }\end{array}$ & $\begin{array}{l}\text { Dos pacientes entrevistados } 81 \% \text { relataram problemas } \\
\text { moderados ou grandes na funcão sexual, } 19 \% \text { na urinária } \\
17 \% \text { na intestinal. Os agravamentos dos sintomas foram } \\
\text { significativamente associados aos niveis mais baixos de } \\
\text { suporte funcional e com menor QV, sendo necessário } \\
\text { intervenções para elevar o nivel de apoio funcional e } \\
\text { melhor QV. }\end{array}$ \\
\hline $\begin{array}{l}\text { Explorar o impacto do } \\
\text { tratamento, o estado } \\
\text { de saúde e as } \\
\text { experiências de } \\
\text { homens com CaP } \\
\text { (CAMERON et al., } \\
\text { 2012). }\end{array}$ & $\begin{array}{l}\text { Prospectivo } \\
73 \\
\text { The European } \\
\text { Qual ity of Life Scale } \\
\text { (EQ-5D) } \\
\text { EPIC } \\
\end{array}$ & $\begin{array}{l}\text { Os escores pós-tratamento mostraram aumento de } \\
\text { problemas nas funções urinária, intestinal e exual. Os } \\
\text { resultados ressaltaram que as funçôes urinária, sexual e a a } \\
\text { dor foram preditores independentes do estado de saúde após } \\
\text { o trat amento. Concluiu -se que o tratamento representa um } \\
\text { desafio no que diz respeito às experiế ncias dos sintomas e } \\
\text { do estado de saúde nos homens. Portanto, as estratégias de } \\
\text { educação para ajudar os homens a lidarem com os seus } \\
\text { sintomas são importantes. }\end{array}$ \\
\hline $\begin{array}{l}\text { Descrever a evolução } \\
\text { temporal e trajetória } \\
\text { de desenvolvimento } \\
\text { dos sintomas urinários } \\
\text { e intestinais relatados } \\
\text { pelos pacientes } \\
\text { dura nte a radioterapia } \\
\text { (CHEN et al., 2012). }\end{array}$ & $\begin{array}{l}100 \\
\text { The validated } \\
\text { Pro state Cancer } \\
\text { Symptom Indices } \\
\text { (PCSI) }\end{array}$ & $\begin{array}{l}\text { Os sintomas urinários foram frequentes no início do estudo } \\
\text { e se agravou durante o tratamento. Até o final da } \\
\text { radioter apia, } 50 \% \text { dos pacientes desenvolveram sintomas } \\
\text { urinários obstrutivos e irritativos; sintomas intestinais } \\
\text { agudos foram menos frequentes. Os resultados fornecem } \\
\text { dados do ponto de vista do paciente, que podem ser usados } \\
\text { pelos médicos durante o processo de tomada de decisão em } \\
\text { relação ao tratamento. }\end{array}$ \\
\hline $\begin{array}{l}\text { Avaliar as trajetórias } \\
\text { de ocorrências, } \\
\text { gravidade e angú stia } \\
\text { dos seis sintomas mais } \\
\text { prevalentes relatados } \\
\text { por pacientes } \\
\text { subm etidos à } \\
\text { radioterapia (KNAPP } \\
\text { et al., 2012). }\end{array}$ & $\begin{array}{l}\text { Prospectivo } \\
\quad 82 \\
\text { Memorial Symptom } \\
\text { Assessment Scale } \\
\text { Escala Karnofsky } \\
\text { Perfo rmance Status } \\
\end{array}$ & $\begin{array}{l}\text { As dimensões dor, falta de energia, sensação de sonolência } \\
\text { e dificuldade de dormir seguiram uma tendência linear } \\
\text { decrescente. Problemas com a mição e diarreia } \\
\text { demonstr aram padrões mais complexos de mudança ao } \\
\text { longo do tempo. Embora os dados longitudinais sobre a dor, } \\
\text { falta de energia, sensação de sonolência e dificuldade em } \\
\text { dormir tenham sido limitados, foram altame nte prevalentes } \\
\text { nos pacientes. }\end{array}$ \\
\hline
\end{tabular}

Quadro 3: Síntese dos estudos que avaliaram com foco na comparação entre modalidades de radiação e de alterações de aumento das doses de radiação.

\begin{tabular}{|c|c|c|}
\hline Objetivos & $\begin{array}{c}\text { Delineame ntos/ } \\
\text { Amostras e } \\
\text { Instrumentos }\end{array}$ & Resultados/Conclusões \\
\hline $\begin{array}{l}\text { Investigar se a QV, } \\
\text { após radioterapia de } \\
\text { intensidade modulada, } \\
\text { melhora através da } \\
\text { redução de margens } \\
\text { do volume alvo, } \\
\text { acompanhando o } \\
\text { tumor em tempo real } \\
\text { (SANDLER et al., } \\
\text { 2010). }\end{array}$ & $\begin{array}{c}\text { Prospectivo } \\
217 \\
\text { EPIC }\end{array}$ & $\begin{array}{l}\text { Os pacientes tratados com margens de redução } \\
\text { apresent aram caracteristicas clinicas menos favoráveis, com } \\
\text { escores pré-tratamento semelhantes ou ligeiramente mais } \\
\text { pobres nas funçōes intestinal, urinária e exexual, do que os } \\
\text { tratados com intensidade modulada. Pacientes tratados com } \\
\text { margens reduzidas e rastreamento do tumor tiveram menos } \\
\text { morbidez relacionada à radioterapia do que os tratados com } \\
\text { margens convencionais. }\end{array}$ \\
\hline $\begin{array}{l}\text { Avaliar o impacto do } \\
\text { aumento da dose de } \\
\text { radioterapia na QV de } \\
\text { pacientes com CaP } \\
\text { (AL-MAMGANI et } \\
\text { al., 2011). }\end{array}$ & $\begin{array}{c}\text { Randomizado } \\
\qquad 300 \\
\text { SF-36 }\end{array}$ & $\begin{array}{l}\text { Os pacientes foram distribuidos aleatoriamente para receber } \\
\text { as doses de } 68 \mathrm{e} 78 \mathrm{~Gy} \text {; a redução na pontuação de QV, em } \\
\text { geral, foi mais pronunciada no grupo da dose mais elevada } \\
\text { do que no da dose mais baixa e na saúde fisica do que na } \\
\text { saúde mental. Em ambas as doses, houve uma diminuição } \\
\text { estatisticamente significativa nos escores de QV ao longo } \\
\text { do tempo. }\end{array}$ \\
\hline $\begin{array}{l}\text { Comparar as } \\
\text { alter açōes na QVRS } \\
\text { após radioterapia de } \\
\text { inte nsidade modulada } \\
\text { e radioterapia } \\
\text { confo rmada } \\
\text { (PINKAWA et al., } \\
\text { 2011). }\end{array}$ & $\begin{array}{c}\text { Prospectivo } \\
362 \\
\text { EPIC }\end{array}$ & $\begin{array}{l}\text { Pacientes com ereções firmes para relações sexuais antes do } \\
\text { tratamento perderam essa capacidade em } 35 \% \text { após a } \\
\text { radioterapia de intensidade modulada e em } 68 \% \text { após } \\
\text { radioter apia conformada. Em ambas as radiações foram } \\
\text { semelhantes as alteraçóes nos outros escor es de QV. As } \\
\text { vantagens encontradas neste estudo foram no alivio mais } \\
\text { rápido das dores durante as evacuações. }\end{array}$ \\
\hline $\begin{array}{l}\text { Avaliar a toxicidade } \\
\text { aguda e a QV de } \\
\text { pacientes com CaP de } \\
\text { alto risco que } \\
\text { receb eram radiação } \\
\text { hipofr acionada e } \\
\text { convenci onalmente } \\
\text { fracionada de } \\
\text { intensidade mod ulada } \\
\text { (NORKUS et al., } \\
\text { 2013). }\end{array}$ & $\begin{array}{c}\text { Randomizado } \\
\qquad 124 \\
\text { EPIC }\end{array}$ & $\begin{array}{l}\text { Houve um pequeno, mas significativo aumento nas reações } \\
\text { agudas na função geniturinária na primeira e segunda } \\
\text { semana da radiação hipofracionada. Todos desenvolveram } \\
\text { toxicidade gastrointestinal e geniturinária no início do } \\
\text { tratamento, os pacientes do grupo de hipofracionamento } \\
\text { recupeneraram mais rápido. Maior tempo de seguimento é } \\
\text { necesssário para determinar a significância dessas } \\
\text { associaçōes com toxicidade tardia. }\end{array}$ \\
\hline
\end{tabular}

\section{Discussão}

Numa visão geral dos estudos fica evidente a preocupação dos autores em identificar preditores que possam afetar positiva ou negativamente a QVRS de pacientes em tratamento de radioterapia, através do uso de instrumentos de medidas gerais e específicas.

Ainda que os estudos tenham sido realizados em momentos e países diferentes, com amostras, modalidades de radiação e instrumentos distintos, os resultados possibilitaram a identificação dos efeitos dos diferentes tipos de radiação e suas consequências na vida dos pacientes e de medidas terapêutica menos impactantes, contribuindo para o planejamento, a execução e avaliação do tratamento radioterápico no cotidiano dos serviços oncológicos, considerando a realidade de cada um.

Os estudos trazem evidências de que o tratamento de radioterapia causa impacto na QVRS dos pacientes, com manifestações de sinais e sintomas de intensidade variada. As funções mais atingidas durante o tratamento e com maior repercussão na QVRS foram a urinária, sexual e intestinal, afetando em média 50\% dos pacientes (HOWLETT et al., 2010; SANDLER et al., 2010; KATZ et al., 2010; QUEENAN et al, 2010; PINKAWA et al., 2011; HOPPE et al., 2012; CARMERON et al., 2012; CHEN et al., 2012; NORKUS et al., 2013; YAMAMOTO et al., 2014; CHENNUPATI et al., 2014). Um estudo, que buscou esclarecer a relação do apoio social com a QVRS, considerou que os efeitos colaterais na função urinária são potencialmente estigmatizantes, sendo a maior preocupação dos pacientes, contribuindo para a diminuição do contato social (QUEENAN et al., 2010). Outros estudos citam que os sinais e sintomas de dor, falta de energia, sensação de sonolência e dificuldade para dormir foram altamente prevalentes nos pacientes e que naqueles com outras doenças crônicas, além do câncer, ocorreram maiores quedas na QVRS durante o tratamento radioterápico instituído (AL-MAMGANI et al., 2011; KNAPP et al., 2012; SCHAAKE et al., 2013).

Ressalta-se que mesmo havendo declínio na QVRS durante o tratamento, os autores que avaliaram os pacientes em diferentes momentos (antes, durante e após o tratamento), consideraram que os efeitos negativos sobre a QVRS são limitados e principalmente temporários (GEINITZ et al., 2010; KATZ et al., 2010; HOPPE et al., 2012; YAMAMOTO et al., 2014; CHENNUPATI et al., 2014).

A avaliação prospectiva da QVRS em modalidades especificas de radiação permite o conhecimento dos sinais e sintomas mais afetados, além da eficácia terapêutica das diferentes técnicas de radiação existentes. O conhecimento da QVRS pode fornecer dados para uma tomada de decisão mais racional, quer para o paciente, quer para uma determinada população em tratamento de radioterapia. A mesma avaliação prospectiva da QRVS de pacientes com longa sobrevivência, após o tratamento, permite obter informações que podem melhorar os procedimentos terapêuticos a serem utilizados em outros pacientes com câncer de próstata com a mesma indicação terapêutica (GEINITZ et al., 2010; KATZ et al., 2010; HOPPE et al., 2012; SCHAAKE et al., 2013; YAMAMOTO et al., 2014; CHENNUPATI et al., 2014).

Avaliar os tipos de radiação e comparar suas diferentes modalidades foram objetivos de quatro estudos ( SANDLER et al., 2010; AL-MAMGANI et al., 2011; PINKAWA et al., 2011; NORKUS et al., 2013), que ressaltaram a possibilidade de uma melhor definição pela equipe médica do tipo de radiação que melhor se enquadra no tratamento dos pacientes, causando menos impacto em sua QVRS, e que as radioterapias conformada, de intensidade modulada e de prótons, são excelentes opções de tratamento para esses pacientes. 


\section{Conclusão}

Esta revisão, apesar das limitações relacionadas ao baixo número de produções encontradas, sem nenhuma no Brasil, possibilitou uma construção sintetizada acerca das evidências sobre os aspectos da QVRS de pacientes com câncer de próstata em tratamento radioterápico. Os resultados evidenciaram que o tratamento de radioterapia causa impacto na QVRS dos pacientes, com manifestações de sinais e sintomas de intensidade variada, principalmente nas funções urinária, sexual e intestinal. Consideraram que em pacientes com outras doenças crônicas, além do câncer, ocorreram maiores quedas na QVRS durante o tratamento radioterápico instituído. Mesmo havendo declínio na QVRS durante o tratamento, os efeitos negativos foram limitados e, principalmente temporários, podendo melhorar com o passar do tempo. A avaliação prospectiva da QVRS em modalidades especificas de radiação permitiu o conhecimento dos sinais e sintomas mais afetados, além da eficácia terapêutica das diferentes técnicas de radiação existentes. Ainda que os estudos tenham sido realizados em momentos e países diferentes, com amostras, modalidades de radiação e instrumentos distintos, os resultados contribuíram para o planejamento, a execução e avaliação do tratamento radioterápico no cotidiano dos serviços oncológicos, considerando a realidade de cada um. Recomendam, ainda, a realização de novas pesquisas de modo a possibilitar a identificação dos efeitos dos diferentes tipos de radiação e suas consequências na vida dos pacientes, com vistas à escolha de medidas terapêuticas menos impactantes.

\section{Referências}

AL-MAMGANI, A. et al. Dose escalation and quality of life in patients with localized prostate cancer treated with radiotherapy: long-term results of the Dutch randomized dose-escalation trial (CKTO 96-10 trial). Int J Radiat Oncol Biol Phys, v. 79, n. 4, p. 1004-1012, 2011.

BRASIL. Ministério da Saúde, Instituto Nacional de Câncer José Alencar Gomes da Silva (INCA). Estimativa 2016: incidência de câncer no Brasil. Rio de Janeiro: Instituto Nacional do Câncer. 2015. Disponível em: http://www.inca. gov.br/estimativa/2016/sintese-de-resultados-comentarios. asp. Acesso em: 30 mar. 2016.

CAMERON, S. et al. A descriptive study of functions, symptoms, and perceived health state after radiotherapy for prostate cancer. Eur J Oncol Nurs, v. 16, n. 3, p. 310-314. 2012.

CHEN, R. C. et al. Patient-reported quality of life during radiation treatment for localized prostate cancer: results from a prospective phase II trial. BJU In, v. 110, n. 11, p. 1690-1695, 2012.

CHENNUPATI, S. K. et al. Late toxicity and quality of life after definitive treatment of prostate cancer: redefining optimal rectal sparing constraints for intensity-modulated radiation therapy. Cancer Med, v. 3, n. 4, p. 954-961, 2014.
CRAMER, J. A.; SPILKER, B. Quality of life and pharmacoeconomics: an introduction. Philadelphia: Lippincot-Raven. 274 p.

DAMIÃO, R. et al. Câncer de próstata. Rev HUPE, v. 14, n. 1, p. 80-86, 2015.

FERLAY, J. et al. Cancer incidence and mortality worldwide: sources, methods and major patterns in Globocan 2012. Int J Cancer, v. 136, n. 5, p. 359-386, 2015.

GEINITZ, P. D. H. et al. Longitudinal analysis of quality of life in patients receiving conformal radiation therapy for prostate cancer. Strahlenther Onk, v. 186, n. 1, p. 46-52, 2010 .

GREIL, R. et al. Retrospective assessment of quality of life and treatment outcome in patients with Hodgkin's disease from 1969 to 1994. Eur J Cancer, v. 35, n. 5, p. 698-706, 1999.

HERR, G. E. et al. Avaliação de conhecimento acerca da doença oncológica e práticas de cuidados com à saúde. Rev Bras Cancerol, v. 59, n. 1, p. 33-41, 2013.

HOPPE, B. S. et al. Erectile function, incontinence, and other quality of life outcomes following proton therapy for prostate cancer in men 60 years old and younger. Cancer, v.118, n.18, p. 4619-4626, 2012.

HOWLETT, K. et al. Changes in sexual function on mood and quality of life in patients undergoing radiation therapy for prostate cancer. Oncol Nurs Forum, v. 37, n. 1, p. 5866,2010 .

JENNEY, M. E. Health-related quality of life, cancer and health care. Eur J Cancer, v. 32, n. 8, p. 1281-1282, 1996.

KATZ, A. J. et al. Stereotactic body radiotherapy for organconfined prostate cancer. BMC Urol, v.10, n. 1, p. 1-5, 2010 .

KNAPP, K. et al. Trajectories and predictors of symptom occurrence, severity, and distress in prostate cancer patients undergoing radiation therapy. J Pain Symptom Manage, v. 44, n. 4, p. 486-507, 2012.

MELNYK, B. M.; FINEOUT-OVERHOLT, E. Evidencebased practice in nursing $\&$ healthcare: a guide to best practice. Philadelphia: Lippincot Williams \& Wilkins, 2011. 599 p.

NORKUS, D. et al. A randomized hypofractionation dose escalation trial for high risk prostate cancer patients: interim analysis of acute toxicity and quality of life in 124 patients. Radiat Oncol, v. 4, n. 8, p. 2-13, 2013.

PAULA, J. M.; SAWADA, N. O. Health-related quality of life of cancer patients undergoing radiotherapy. Rev Rene, v. 16, n. 1, p. 106-113, 2015. 
PINKAWA, M. et al. Combination of dose escalation with technological advances (intensity-modulated and imageguided radiotherapy) is not associated with increased morbidity for patients with prostate cancer. Strahlenther Onkol, v. 187, n. 8, p. 479-484, 2011.

QUEENAN, J. A. et al. Social support and quality of life of prostate cancer patients after radiotherapy treatment. Eur J Cancer Care, v. 19, n. 2, p. 251-259, 2010.

SALVAJOLI, J. V.; SALVAJOLI, B. P. O papel da radioterapia no tratamento do câncer: avanços e desafios. Rev Onco\&, v. 13, n. 3, p. 32-36, 2012.

SANDLER, H. M. et al. Reduction in patient-reported acute morbidity in prostate cancer patients treated with 81-Gy Intensity-modulated radiotherapy using reduced planning target volume margins and electromagnetic tracking: assessing the impact of margin reduction study. Urology, v. 75, n. 5, p. 1004-1008, 2010.

SCHAAKE, W. et al. Quality of life among prostate cancer patients: A prospective longitudinal population-based study. Radiother Oncol, v. 108, n. 2, p. 299-305, 2013.

SCIARRA, A. et al. Predictors for response to intermittent androgen deprivation (IAD) in prostate cancer cases with biochemical progression after surgery. Urol Onco, v. 31, n. 5, p. 607-614, 2013.

SOUZA, M. T.; SILVA, M. D.; CARVALHO, R. Revisão integrativa: o que é e como fazer. Einstein, v. 8, n. 1, p. 102-106, 2010.

TEIXEIRA, E. et al. Integrative literature review step-bystep \& convergences with other methods of review. Rev Enferm UFPI, v. 2, n. 5, p. 3-7, 2013.

YAMAMOTO, S. et al. Longitudinal change in healthrelated quality of life after intensity-modulated radiation monotherapy for clinically localized prostate cancer. Qual Life Res, v. 23, n. 5, p. 1641-1650, 2014. 